\title{
Compensation of hologram distortion by controlling defocus component in reference beam wavefront for angle multiplexed holograms
}

T. Muroi

muroi.t-hc@nhk.or.jp

\section{N. Kinoshita}

\section{N. Ishii}

K. Kamijo

Y. Kawata

\section{H. Kikuchi}

Holographic memory has the potential to function as a recording system with a large capacity and high data-transfer-rate. Photopolymer materials are typically used as a write-once recording medium. When holograms are recorded on this medium, they can distort due to shrinkage or expansion of the materials, which degrades the reconstructed image and causes a higher bit error rate (bER) of the reproduced data. We propose optically compensating for hologram distortion by controlling aberration components in the reference beam wavefront while reproducing data, thereby improving the reproduced data quality. First, we investigated the relation between each aberration component of the reference beam and the signal to noise ratio (SNR) of the reproduced data using numerical simulation and found that horizontal tilt and the defocus component affect the SNR. Next, we experimentally evaluated the reproduced data by controlling the defocus component in the reference beam and found that the bER of the reproduced data could be decreased by controlling the defocus center with respect to the hologram position and phase modulation depth of the defocus component. Then, we investigated a practical control method of the defocus component using an evaluation value similar to the definition of the SNR for actual data reproduction from holograms. Using a defocus controlled wavefront enabled us to decrease the bER from $3.54 \times 10^{-3}$ with a plane wave to $3.14 \times 10^{-4}$. We also investigated how to reduce the bERs of reproduced data in angle multiplexed holograms. By using a defocus controlled wavefront to compensate for hologram distortion on the 40th data page in 80-page angle multiplexed holograms, the bERs of all pages could be decreased to less than $1 \times 10^{-3}$. We showed that controlling the defocus component is an effective way to compensate for hologram distortion and to decrease the bER of reproduced data in holographic memory.

[DOI: http://dx.doi.org/10.2971/jeos.2013.13080]

Keywords: Holographic memory, angle multiplexing, hologram distortion, optical compensation, defocus component

\section{INTRODUCTION}

Holographic memory has been extensively studied for its potential use a recording system with a large capacity and high data transfer rate. As a data format, holographic memory uses a two dimensional data array called data pages. Since different data pages can be recorded at the same position on a medium, high density data recording is expected, which should lead to a large capacity memory. In addition, a high data transfer rate is expected because a lot of data on one page can be recorded and reproduced at the same time.

Photopolymer materials are currently a popular choice as a write-once holographic recording medium, mostly due to their large $\mathrm{M} / \#$ and high stability. However, they do shrink slightly due to photopolymerization when a laser beam is irradiated to the medium during data recording and when the light for post-curing to store recorded data is irradiated [1]. They can also shrink or expand when the ambient temperature of the medium changes between recording and reproducing [2]-[4]. Such shrinkage and expansion causes distortion in the holograms. When images are reconstructed from these distorted holograms using a reference beam that has the same conditions as those while recording, some areas in the reconstructed images become dark and the data from these areas cannot be reproduced. As a result, the signal to noise ratio (SNR) of the reproduced data decreases and the bit error rate (bER) increases. Potential methods to optically compen- 
sate for such hologram distortions include adjusting the incident angle, controlling the wavelength, and optimizing the wavefront of the reference beam while reproducing [2]-[6]. However, methods that combine adjusting the incident angle with controlling the wavelength of the reference beam cannot sufficiently compensate for hologram distortion when a high NA object lens is used to record data with high density. In contrast, a method that combines adjusting the incident angle with optimizing the wavefront of the reference beam can compensate for distortion and improve the bER of the reproduced data even if a high NA object lens is used [5, 6]. However, the problem with this latter approach is that it is difficult to individually obtain a satisfactory wavefront to compensate for complex hologram distortion for each data page while real-time data reproduction is performed in multiplexed holograms. Incidentally, real-time aberration compensation by the beam has been successfully used to detect bits in Blu-Ray disc drivers. To compensate for aberration correctly, the wavefront is divided into aberration components-defocus, astigmatism, and coma-that are independently controlled and compensate for any aberration following bits on a fast rotating disk. What this means is that if the responsibility for compensating was divided among each aberration component of the reference beam wavefront in holographic memory, real-time compensation following data reproduction would be possible the same as the aberration compensation used in optical disk drivers.

In this paper, we investigate the relation between aberration components of the reference beam wavefront while reproducing data and the SNR of the reproduced data and identify which aberration components improve the SNR. In addition, we show that the bER of the reproduced data can be decreased by controlling these components.

\section{RELATION BETWEEN ABERRATION COMPONENTS OF WAVEFRONT AND SNR OF REPRODUCED DATA}

First, we analyzed the optical compensation of hologram distortion and the SNR of the reproduced data using numerical simulation when any type of reference beam wavefront enters the hologram while reproducing. Since the main shrinkage of the medium occurs in the direction normalized to the medium surface, we only consider hologram distortion in this direction. This numerical simulation technique is based on the scalar diffraction theory. The medium was divided into a number of thin layers to implement the volume property of the recording. Each diffracted wave in the layer is calculated and all diffracted waves are added, leading to the final diffraction beam. This simulation has been limited to cases in which the recording medium was located normal to the signal beam axis $[7,8]$. However, in angle multiplexed holograms using the high NA object lens to increase data recording density, since the convergent angle of the signal beam becomes large, a configuration in which the medium is tilted with respect to the signal beam axis is required for irradiating the reference beam at various incident angles. Therefore, we employed a numerical simulation that includes a rotational transforma- tion of the wave field and can deal with the tilted medium configuration [9].

When recorded holograms do not have any distortion, the reference beam wavefront for reproducing is a plane wave. However, when the holograms do have distortion, the reference beam wavefront to optically compensate for distortion is not a plane wave [5] and must therefore be expressed as a combination of aberration components. We used Zernike polynomials as the expression of aberration components. The relation between order and equation in Zernike polynomials is shown in Table 1 . The first and second orders show horizontal and vertical tilts, the third shows defocus, the fourth and fifth show 3rd order astigmatism aberration, the sixth and seventh show 3rd order coma aberration, the eighth shows 3rd order spherical aberration, and the ninth and above show their higher harmonics. In this simulation, first, we calculated the SNRs of the reproduced data with changing the coefficient value of the first order in the Zernike polynomials of the reference beam and obtained the coefficient value at maximum SNR. The SNR of the reproduced data is expressed by

$$
\mathrm{SNR} \equiv 20 \log \left(\frac{\mu_{1}-\mu_{0}}{\sqrt{\sigma_{1}^{2}+\sigma_{0}^{2}}}\right)
$$

where $\sigma$ is standard deviation, $\mu$ is the mean of the brightness of bits, and subscripts 0 and 1 are bits 0 and 1 , respectively. Next, under the condition where the coefficient value of the first order is at a maximum SNR, we calculate the SNR with changing the coefficient value of the second order and obtain the coefficient value at maximum SNR. We then repeat this process with increasing order in the Zernike polynomials. The parameters in this numerical simulation were a recording layer thickness of $1 \mathrm{~mm}$, a shrinkage ratio of $0.105 \%$ and hologram distortion occurring in only the direction normalized to the medium surface. The wavelength of the laser was $532 \mathrm{~nm}$, the reference beam angle with respect to the signal beam axis was $70^{\circ}$, the angle between the direction normalized to the medium surface and the signal beam axis was $15^{\circ}$, the focal length of the object lens was $11.0 \mathrm{~mm}$, and the image pixel pitch was $31.2 \mu \mathrm{m}$. Figure 1 shows the simulation results of the reconstructed image. Figure 1(a) shows the reconstructed image when the reference beam angle and wavefront of the plane wave while reproducing were the same as those while recording. There were dark areas from the center to the upper center in the image due to hologram distortion caused by shrinkage, and the reproduced data could not be obtained from these areas. Figure 1(b) shows the image when the coefficient value

\begin{tabular}{|c|l|l|}
\hline Order & Equation & Aberration name \\
\hline 1 & $\rho \cos (\theta)$ & Tilt at $0^{\circ}$ \\
2 & $\rho \sin (\theta)$ & Tilt at $90^{\circ}$ \\
3 & $2 \rho^{2}-1$ & Defocus \\
4 & $\rho^{2} \cos (2 \theta)$ & $3^{\text {rd }}$ order astigmatism at $0^{\circ}$ \\
5 & $\rho^{2} \sin (2 \theta)$ & $3^{\text {rd } \text { order astigmatism at } 45^{\circ}}$ \\
6 & $\left(3 \rho^{2}-2\right) \rho \cos (\theta)$ & $3^{\text {rd } \text { order coma at } 0^{\circ}}$ \\
7 & $\left(3 \rho^{2}-2\right) \rho \sin (\theta)$ & $3^{\text {rd } \text { order coma at } 90^{\circ}}$ \\
8 & $6 \rho^{4}-6 \rho^{2}+1$ & $3^{\text {rd } \text { order spherical aberration }}$ \\
\hline
\end{tabular}

TABLE 1 Zernike polynomials 
of the first order in the Zernike polynomials was controlled to be a maximum SNR. The dark areas in the image improved, but there were still some remaining at the lower left and right sides of the image. Figure 1(c) shows the image when the coefficient values up to the sixth order were controlled to be maximum SNRs. The reconstructed image was clearly improved and data could be obtained from the entire reconstructed image. The hologram distortion is symmetric with respect to the plane defined by the signal and reference beams. However, hologram distortions on the plane and out of the plane are a little different because incident angle of the signal beam is different due to focusing by the object lens. Therefore, the high order of the Zernike polynomials gives a little influence to the compensation. Figure 2 shows the maximum SNRs as a function of order in Zernike polynomials. The SNR is almost completely saturated over the fourth order. This result indicates that control of less than the third order in Zernike polynomials, especially the first and third orders-namely, the horizontal tilt and defocus components-can significantly influence the compensation of hologram distortion and improve the SNR of the reproduced data.

\section{COMPENSATION OF HOLOGRAM DISTORTION BY CONTROLLING DEFOCUS COMPONENT}

\subsection{Phase conjugated holographic recording/reproducing optical setup with defocus component control}

Figure 3 shows a holographic recording/reproducing optical setup that can control the horizontal and vertical tilts and the defocus component in the reference beam. This optical setup is a phase conjugation type that can cancel object lens aberration [10]. The laser beam is expanded and divided into signal and reference beams with a polarizing beam splitter (PBS) and the signal beam is amplitude modulated with an amplitude modulation spatial light modulator (AM-SLM) that displays the data pages. The pixel pitch of the AM-SLM is $10.4 \mu \mathrm{m}$, which is equal to the pixel pitch of the data page image. The signal beam with data is focused on the medium by the object lens. The NA of the lens is 0.85 and focal length is $11.0 \mathrm{~mm}$. The reference beam is divided into two beams: one for recording and the other for reproducing. The incident angles of the reference beam while recording as it enters the same position of the medium is controlled by a galvanometer mirror (GM) and relay lens. The signal beam and the reference beam while recording enter the medium on the same side. Data are recorded by interference fringes between the signal beam with data and the reference beam. In contrast, in the path of the reference beam while reproducing, a phase modulation spatial light modulator (PM-SLM) is set to control the wavefront of the beam. The pixel size of PM-SLM is $10.4 \mu \mathrm{m}$ and the maximum phase modulation depth is $4 \pi$ in each pixel at the wavelength of $532 \mathrm{~nm}$. The PM-SLM can control any type of aberration. The wavefront controlled reference beam enters the opposite side of the medium than it does while recording after the horizontal and vertical incident angles of the beam are adjusted by a 2-axis GM. These horizontal and vertical tilts controlled by the GM correspond to controls of the first

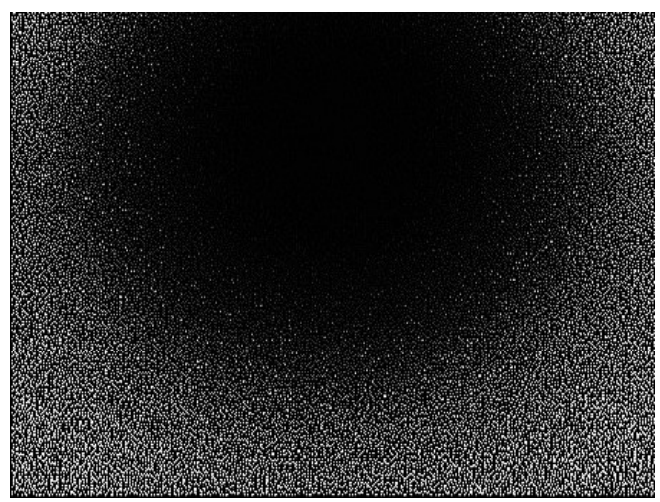

(a) Plane wave without control.

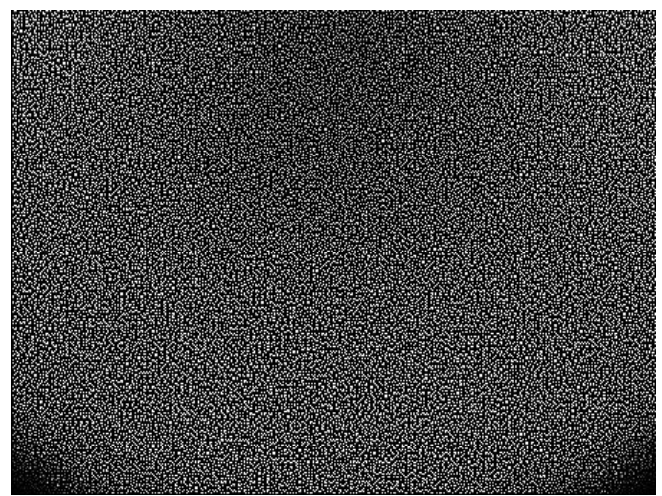

(b) Plane wave with horizontal tilt control

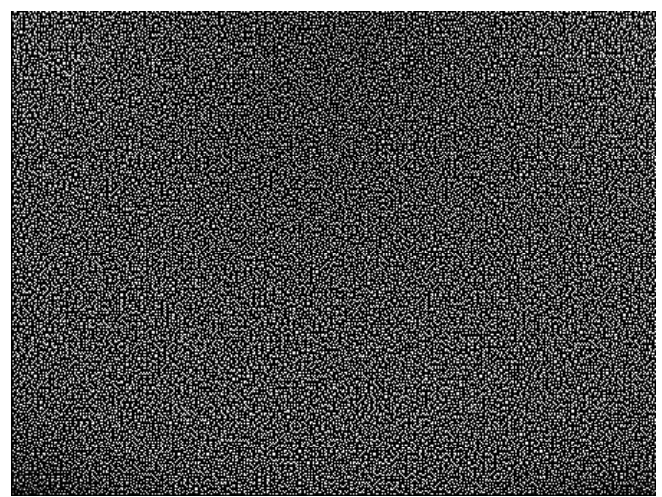

(c) Wavefront with control between first and sixth order aberration components

FIG. 1 Simulation results of reconstructed image.

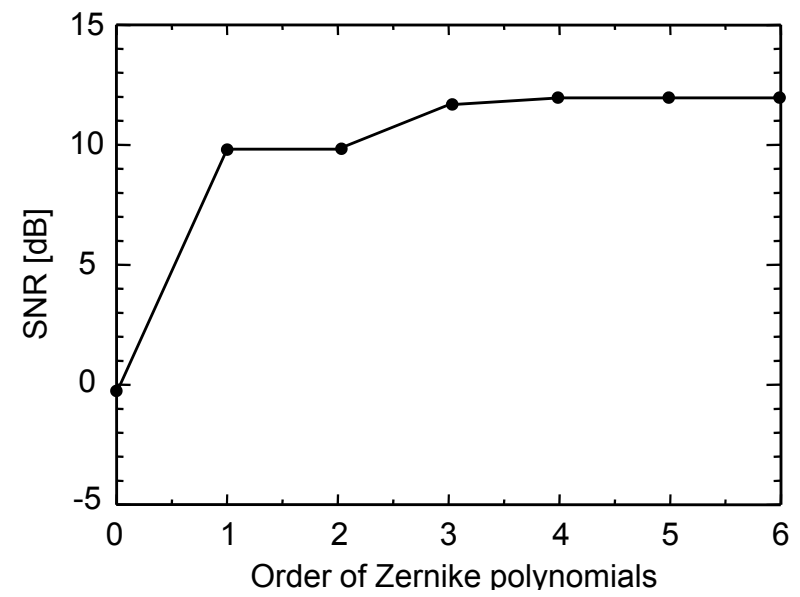

FIG. 2 Simulation result of increasing the SNR by controlling aberration components of reference beam. 


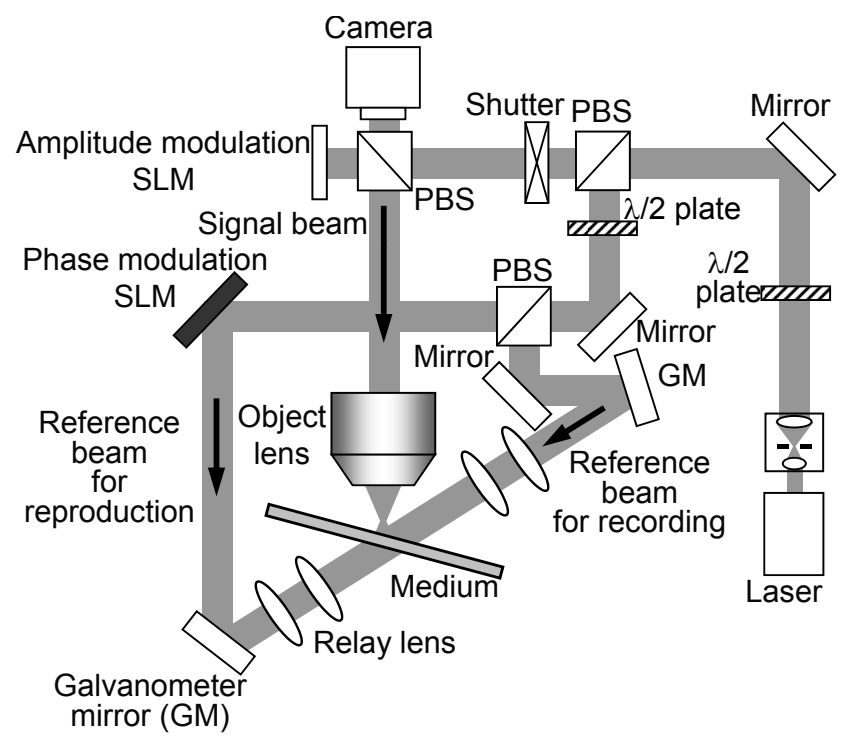

FIG. 3 Optical setup of holographic memory with wavefront control.

and second order of Zernike polynomials while the defocus component control by PM-SLM corresponds to control of the third order of Zernike polynomials. The reconstructed beam diffracted by holograms goes through the object lens and the PBS before arriving at the camera. Since the signal and the reconstructed beams go through the same object lens but in the opposite direction, the lens aberration can be canceled even if the high NA lens is used. The reproduced data can then be obtained by capturing the reconstructed image.

\subsection{Reduction of bER by controlling defocus component}

We experimentally investigated compensation of hologram distortion by controlling the horizontal and vertical tilts and the defocus component using the holographic recording/reproducing optical setup shown in Figure 3. We recorded 50 data pages of holograms with angle multiplexing. The incident angle of the reference beam with respect to the signal beam axis was between $63.75^{\circ}$ and $76.25^{\circ}$ and the angle gap between neighboring data pages was $0.25^{\circ}$. The recording medium was made by Nippon Steel \& Sumikin Chemical Co., Ltd. We used a medium that had a large shrinkage ratio in order to investigate the effect of wavefront compensation. The thickness of photopolymer layer in the medium is $1 \mathrm{~mm}$. The wavefront of the reference beam while recording was a plane wave. After recording the data and conducting post curing on the medium, data were reproduced using the reference beam with a plane wave. Figure 4(a) shows the reconstructed image of the first hologram. The horizontal and vertical angles of the reference beam were adjusted with the 2-axis GM to have a lower bER. The medium slightly shrinks heterogeneously in addition to the shrinkage in the direction normalized to the medium surface in the actual data recording. This shrinkage causes hologram distortion in various direction. If there are no angle adjustment with the 2-axis GM, which means that incident angle during reproduction is the same as that during recording, the reconstructed image cannot be obtained at all. Therefore, the 2-axis angle adjustments are needed. However, the dark areas in the upper center, lower right, and left side

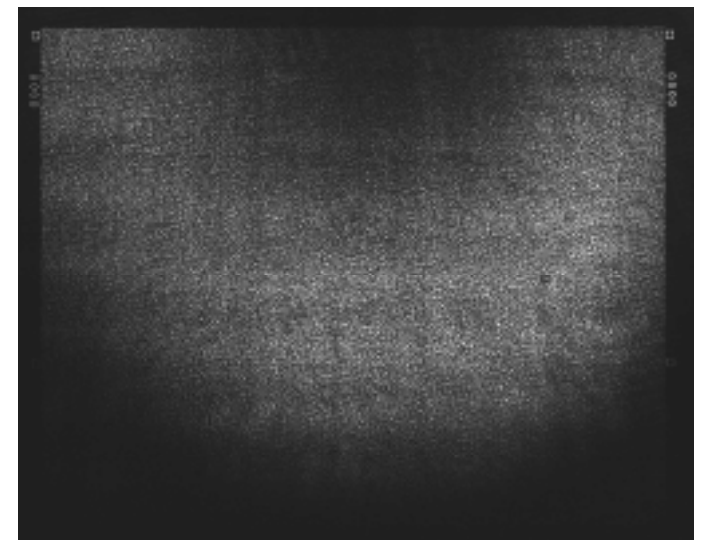

(a) Plane wave with tilt controlled to lower bER

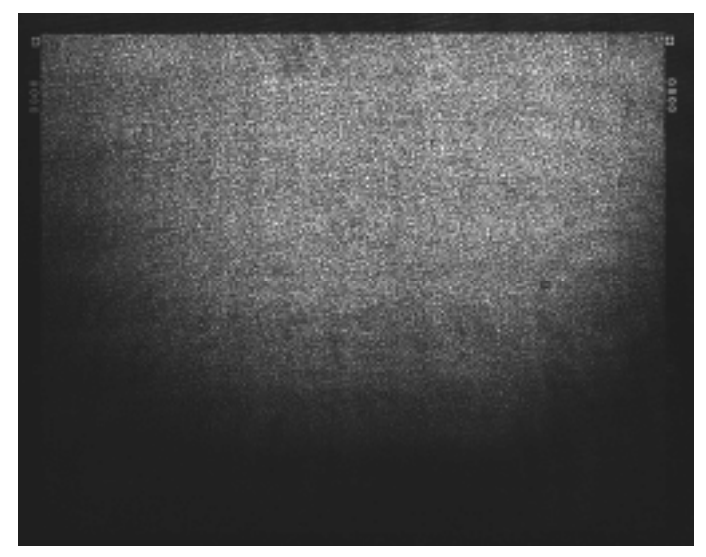

(b) Plane wave with tilt controlled to reduce upper dark area in (a)

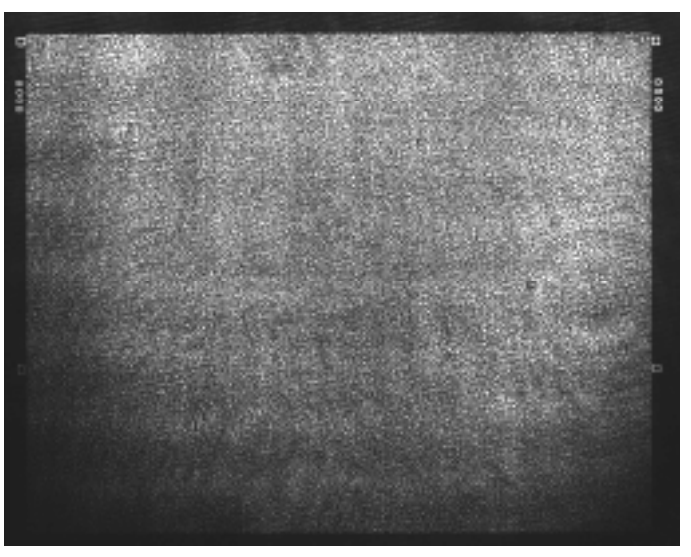

(c) Defocus controlled wavefront

FIG. 4 Improvement of reconstructed images using defocus controlled wavefront for compensation.

of the image remain. The bER of the reproduced data was $4.0 \times 10^{-2}$.

We reduced the bER of the reproduced data shown in Figure 4(a) by compensating for the hologram distortion. First, in order to reduce the upper center dark area in the image, the reference beam angle was adjusted with the GM. As shown in Figure 4(b), the upper center dark area decreased. However, the lower right and left side dark areas increased. Next, we controlled the defocus component of the reference beam during reproduction by using the PM-SLM. Figure 5(a) shows the signal displayed on the PM-SLM to control the defocus. The parameters were the center position and the phase modulation depth of defocus. First, the center position was investigated. The signal to the PM-SLM was controlled so as to put 


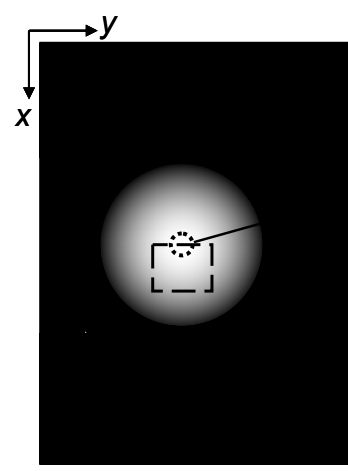

(a) Signal on SLM the center of the defocus position at the center of the upper line of the reproduced data area. Namely, as shown in Figure 5 , the center of the defocus position (dotted circle in (a)) corresponds to the center on the upper line of the reproduced data area (dotted circle in (b)). The $(x, y)$ coordinates of the center position on the PM-SLM were about $(680,460)$. The dotted rectangle area in Figure 5(a) corresponds to the data area in the hologram, and the coordinates of the four corners on the PM-SLM were $(680,400),(680,520),(780,390)$, and $(780,530)$, respectively. Figure 6(a) plots the bER as a function of the horizontal position of the center. The vertical position was 680 and the coefficient of the third order in Zernike polynomials that relates to the phase modulation depth of the defocus was -0.0025 . When the horizontal position was moved to the left (horizontal coordinates decrease), the bER increased. This is because the dark areas at the lower left and right side of the reconstructed image increased, and in some cases, the bit data of the upper left side got blurred. Also, when the horizontal position was moved to the right (horizontal coordinates increase), the bER increased. This is because the dark areas at the lower left and right sides of the reconstructed image increased. Next, the center of the defocus position was moved vertically from the position $(680,440)$, where the bER was lowest in Figure 6(a). Figure 6(b) shows the bER as a function of the vertical position of the center. When the vertical position was moved upward (vertical coordinates decrease), the bER increased because the dark areas at lower left increased. When the vertical position was moved downward (vertical coordinates increase), the bER increased because the dark areas at lower right increased. Finally, the modulation depth of the defocus moved. Figure 6(c) plots the bER as a function of the coefficient of the third order in Zernike polynomials. The bER decreases as the coefficient decreases from 0 to -0.0023 . When the coefficient is -0.0023 , the bER is at a minimum and the dark area in the reconstructed image is also at a minimum. As the coefficient decreases further, the bER increases again and the dark area in the image increases. Figure 4(c) shows the reconstructed image in which the dark area in the image is at a minimum. In this case, the maximum phase deference in the hologram was about $\pi$. The bER of the reproduced data could be decreased to $1.7 \times 10^{-3}$. Although the vertical position corresponds to the upper line of the reconstructed data area in this case, we guess that the vertical position does not always correspond to the upper line of the data are because the center position and modulation depth of the defocus depends on an amount of shrinkage of the medium. However, these re-

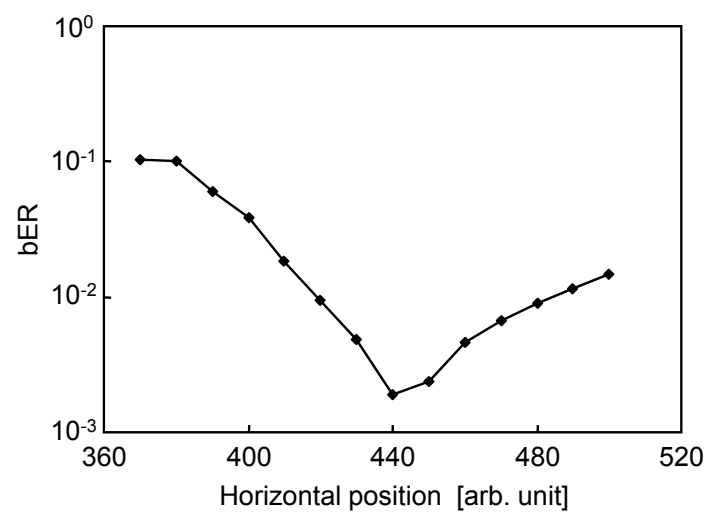

(a) Horizontal position

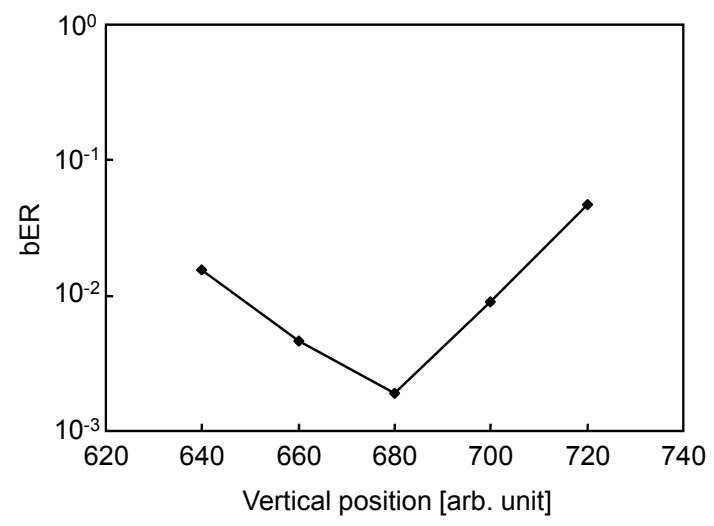

(b) Vertical position

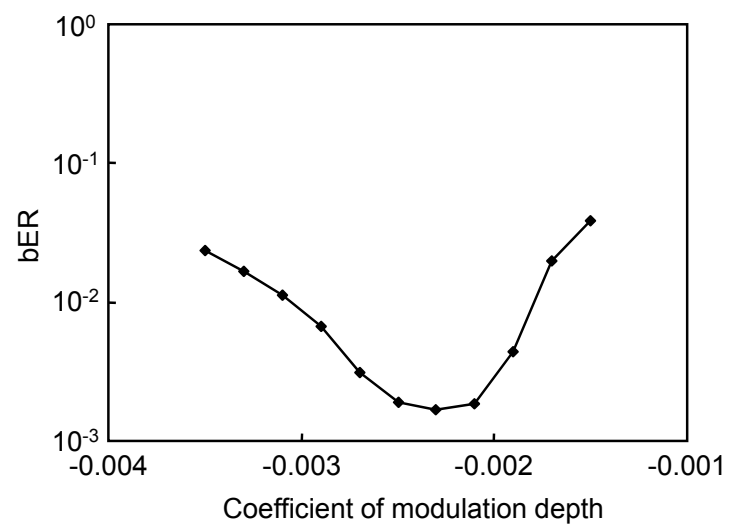

(c) Coefficient of phase modulation depth

FIC. 6 bER as a function of defocus center position and coefficient of phase modulation depth of defocus.

sults indicate that the bER can be decreased by controlling the center position and phase modulation depth of the defocus component in the reference beam wavefront.

\section{PRACTICAL CONTROL METHOD OF DEFOCUS COMPONENT IN ACTUAL DATA REPRODUCTION}

\subsection{Practical control method of defocus component}

In the previous section, we experimentally investigated the relation between the defocus component in a reference beam and the bER of the reproduced data and found that the bER could be decreased by controlling the defocus component. In this experiment, we calculated the bER using the original data 
to be recorded on the medium and the reproduced data because the original data was known in advance. However, in practical usage, the bER cannot be calculated because we do not know the original data. We therefore developed a practical method of controlling the defocus component that is effective for actually reproducing data from holograms.

In our previous studies, we showed that adaptive optics with a genetic algorithm can optimize the wavefront of the reference beam to compensate for hologram distortion and improve the bER of the reproduced data $[5,6]$. With this type of adaptive optics, the evaluation value expressed by

$$
\mathrm{EV} \equiv\left(\mu_{1}-\mu_{0}\right) / \sqrt{\left(c_{1}^{2}+c_{0}^{2}\right)}
$$

is used to evaluate the reproduced data quality [6]. Here, $\mu$ is the mean of the brightness of the bits and $c$ is the coefficient of variation, which is the standard deviation $\sigma$ normalized to the mean brightness and expressed by $\sigma / \mu$. Subscripts 0 and 1 represent bit 0 and 1 , respectively. These values were obtained from the reproduced data. This evaluation value is similar to the definition of SNR represented by Eq. (1). We use this evaluation value to control the defocus component.

In the reconstructed image that has a dark area at the lower left and right due to hologram distortion, first, we obtain the horizontal position of the defocus center. We moved the center position horizontally from the upper left to the upper right along the upper edge of the reproduced data area, captured the reconstructed image, and calculated the evaluation value represented by Eq. (2). Figure 7(a) plots the evaluation value as a function of the horizontal position of the defocus center. When the coordination of the horizontal position on the PMSLM was 460 , the evaluation value was at a maximum. Next, at the horizontal center position at which the evaluation value was at a maximum, we moved the center position vertically and evaluated the value again. Figure $7(\mathrm{~b})$ plots the evaluation value as a function of the vertical position of the defocus center. When the coordination of the vertical position on the PMSLM was 675, the evaluation value was at a maximum. After obtaining the defocus center position, the phase modulation depth was optimized using the evaluation value. Figure 7(c) plots the evaluation value as a function of the coefficient value of the third order in Zernike polynomials that expresses the phase modulation depth of the defocus component. When the coefficient is -0.0011 , the evaluation value was at a maximum. We verified the bER reduction of the reproduced data using the reference beam with this controlled wavefront. The dark areas at the lower left and right in the reconstructed image were reduced and the bER of the reproduced data was decreased from $3.54 \times 10^{-3}$ with a plane wave to $3.14 \times 10^{-4}$. This result indicates that the proposed control method is an effective means of decreasing the bER of the reproduced data in actual data reproduction in holographic memory.

\subsection{Reduction of bER in angle multiplexed holograms}

We investigated the compensation of hologram distortion and reduction of the bER of reproduced data by controlling the defocus component of the reference beam in angle multiplexed

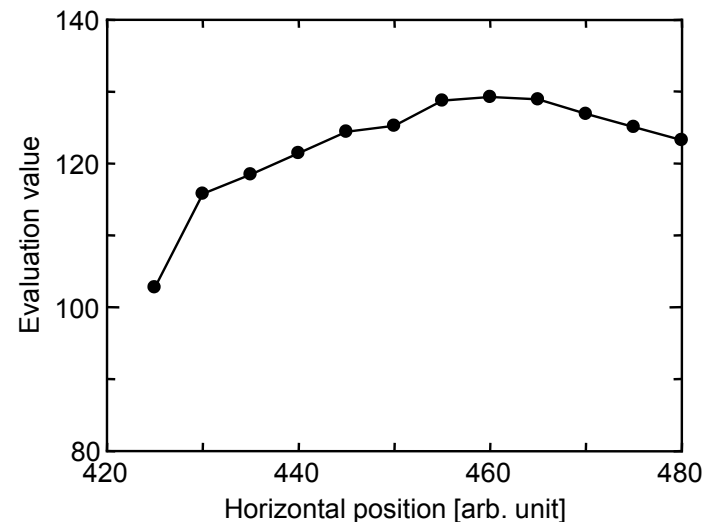

(a) Horizontal position

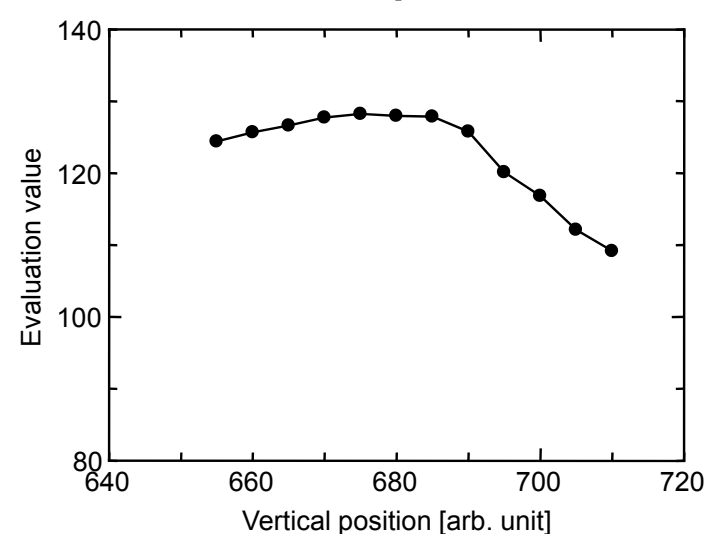

(b) Vertical position

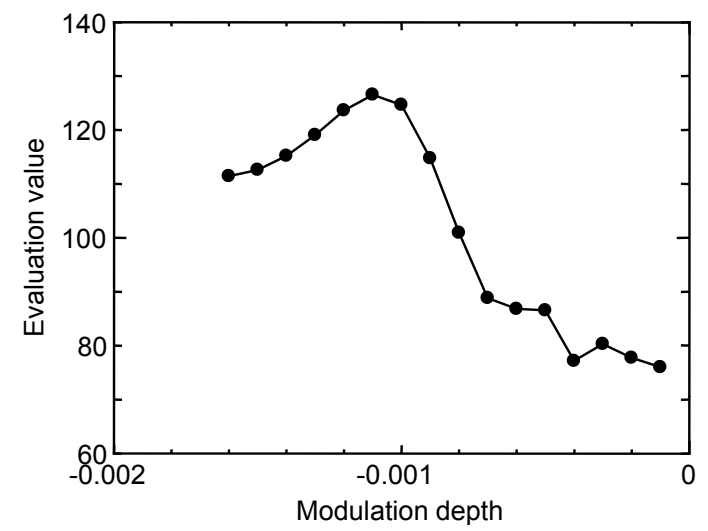

(c) Coefficient of phase modulation depth

FIG. 7 Evaluation value as a function of defocus center position and phase modulation depth of defocus

holograms. Figure 8 plots the bERs of the reproduced data when 80 data pages are reproduced from angle multiplexed holograms. The reference beam angle with respect to the signal beam axis was between $57.5^{\circ}$ and $77.5^{\circ}$ and the angle gap between neighboring data pages was $0.25^{\circ}$. The white dots in the figure indicate the bERs when the plane wave was used for reproducing. Horizontal and vertical tilts were adjusted by the 2-axis GM to make bERs a minimum. The bERs of the reproduced data were more than $1 \times 10^{-2}$ on all data pages. In angle multiplexed holograms, since the difference of hologram distortion between several neighboring data pages is not very big, the wavefront to compensate for hologram distortion on any page can compensate for hologram distortion on several neighboring data pages [6]. Therefore, we were able to control the defocus component to compensate for hologram distortion on page number 39 (the 40th data page), which is directly in the middle of the 80 data pages in angle multiplexed 


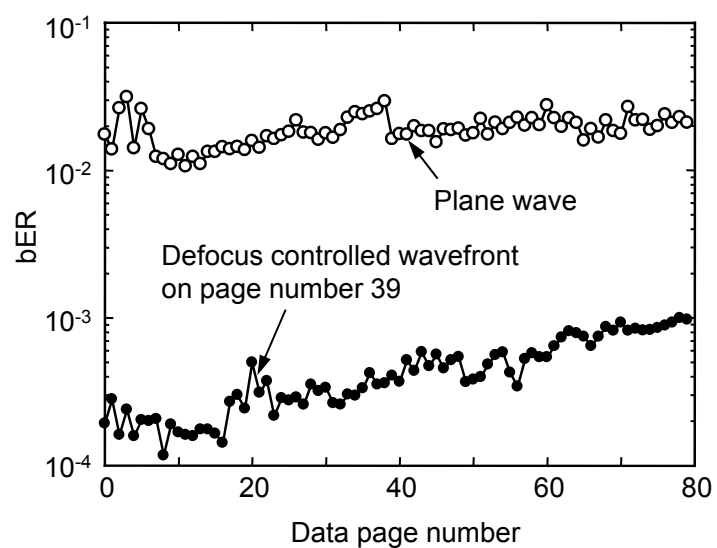

FIG. 8 Reduction of bER of reproduced data by defocus controlled wavefront on 40th data page in angle multiplexed hologram.

holograms. The black dots in Figure 8 show the bERs of the reproduced data when the defocus controlled wavefront on page number 39 is used for reproducing all data pages. Horizontal and vertical tilts were also adjusted to make the bER a minimum. Hologram distortion could be compensated and the bERs could be decreased to less than $1 \times 10^{-3}$ on all data pages.

\section{CONCLUSION}

In holographic memory, when holograms are recorded on a medium that consists of photopolymer materials, the holograms can distort due to shrinkage or expansion, which causes the reproduced data to have a higher bER. We therefore investigated optical compensation of hologram distortion by controlling aberration components in the reference beam wavefront during reproduction to improve the reproduced data quality. First, we investigated the relation between each aberration component of the reference beam and the SNR of the reproduced data using numerical simulation. Results showed that horizontal tilt and the defocus component both affect the SNR. Next, we experimentally evaluated the reproduced data by controlling the defocus component in the reference beam and found that the bER of the reproduced data could be decreased by controlling the defocus center with respect to the hologram position and phase modulation depth of the defocus component. Then, we tested the practical control method of the defocus component using an evaluation value similar to the definition of the SNR for actual data reproduction from holograms and demonstrated that this method is an effective means of decreasing the bER of the reproduced data. We also investigated how to reduce the bERs of reproduced data in angle multiplexed holograms. By using a defocus controlled wavefront to compensate for hologram distortion on the 40th data page of an 80-page angle multiplexed hologram, the bERs of all pages could be decreased. Controlling the defocus component of the reference beam is an effective means of compensating for hologram distortion and decreasing the bER of reproduced data in angle multiplexed holograms.

\section{ACKNOWLEDGEMENTS}

We are grateful to Nippon Steel \& Sumikin Chemical Co., Ltd. for providing us with their photopolymer recording medium for the experiments and for giving us helpful advice on its use.

\section{References}

[1] L. Dhar, M. G. Schnoes, T. L. Wysocki, H. Bair, M. Schilling, and C. Boyd, "Temperature-induced changes in photopolymer volume holograms," Appl. Phys. Lett. 73, 1337-1339 (1998).

[2] M. Toishi, T. Tanaka, M. Sugiki, and K. Watanabe, "Improvement in temperature tolerance of holographic data storage using wavelength tunable laser," Jpn. J. Appl. Phys. 45, 1297-1304 (2006).

[3] T. Tanaka, and K. Watanabe, "Analytical solution to compensate for thermal expansion change in photopolymer volume holograms using a tunable laser," Appl. Opt. 47, 776-783 (2008).

[4] T. Muroi, N. Kinoshita, N. Ishii, K. Kamijo, and N. Shimidzu, “Compensation of interference fringe distortion due to temperature variation in holographic data storage," Jpn. J. Appl. Phys. 49, 08KD03 (2010).

[5] T. Muroi, N. Kinoshita, N. Ishii, K. Kamijo, and N. Shimidzu, "Optical compensation of distorted data image caused by interference fringe distortion in holographic data storage," Appl. 0pt. 48, 3681-3690 (2009).

[6] T. Muroi, N. Kinoshita, N. Ishii, K. Kamijo, H. Kikuchi, Y. Kawata, and N. Shimidzu, "Optical compensation of hologram distortion avoiding interpage crosstalk on reconstructed image in anglemultiplexed holograms," Appl. Opt. 50, 5700-5709 (2011).

[7] M. Miura, 0. Matoba, K. Nitta, and T. Yoshimura, "Image-based numerical evaluation techniques in volue holographic memory systems," J. Opt. Soc. Am. B 24, 792-798 (2007).

[8] Y. Yonetani, K. Nitta, and O. Matoba, "Numerical evaluation of angular multiplexing in reflection-type holographic data storage in photopolymer with shrinkage," Appl. Opt. 49, 694-700 (2010).

[9] N. Kinoshita, T. Muroi, N. Ishii, K. Kamijo, H. Kikuchi, N. Shimidzu, and 0 . Matoba, "Half-data-page insertion method for increasing recording density in angular multiplexing holographic memory," Appl. Opt. 50, 2361-2369 (2011).

[10] J. Ashley, M.-P Bernal, G. W. Burr, H. Coufal, H. Guenther, J. A. Hoffnagle, C. M. Jefferson, B. Marcus, R. M. Macfarlane, R. M. Shelby, and G. T. Sincerbox, "Holographic data storage technology," IBM J. Res. Develop. 44, 341-368 (2000). 